Short Communication

\title{
Preparation of Electrostatic Spinning Composite Film Loaded with Polyvinylpyrrolidone for the Detection of Free Radicals in Polluted Air
}

Guoying Wang ${ }^{1}$, Yanrong Liu ${ }^{1}$, Qiuping Zhao ${ }^{1, *}$, Xuefu Chen ${ }^{1}$, Shengxue Hu ${ }^{2}$, Lan Li $^{1}$, Xia Jiang ${ }^{1}$, Chao Liul, Shiming Jia ${ }^{1}$, Haoqi Tian ${ }^{1}$, Yucan Dong ${ }^{1}$, Peng Zhang ${ }^{1}$, Hua Mal, Gaofeng Shi,

${ }^{1}$ School of Petrochemical Engineering, Lanzhou University of Technology, Lanzhou, China

${ }^{2}$ PetroChina Fushun petrochemical Company Catalyst Plant, Fushun, China

*E-mail: gaofengshi_lzh@163.com, wangguoying@lut.cn

doi: $10.20964 / 2018.12 .06$

Received: 8 July 2018 / Accepted: 23 September 2018 / Published: 5 November 2018

An electrospinning system was used to elongate the prepared radical-scavenging antioxidant compounds into a fibrous-structured nanomaterial under the action of electrostatic force, and its structure was characterized by scanning electron microscopy. The trapping performance of the electrospun membrane was evaluated by comparing the sampling results of two membranes, and the integral peak areas were used for the detection of free radicals also obtained. The experimental results show that the electrospun membrane can effectively trap free radicals in polluted air, and the changes in the integral peak areas of free radicals are related to volatile organic compounds, particulate matters, sulphur dioxide, nitrogen dioxide, carbon monooxide, ozone, sampling time, temperature, and humidity. Subsequently, the nanomaterial was used for outfield sampling, and the sampling performance was measured using a fluorescence spectrophotometer.

Keywords: Air pollutants, Active molecules, Detection of free radicals, Electrospun membrane, Fluorescence spectrophotometer

\section{FULL TEXT}

(C) 2018 The Authors. Published by ESG (www.electrochemsci.org). This article is an open access article distributed under the terms and conditions of the Creative Commons Attribution license (http://creativecommons.org/licenses/by/4.0/). 